\title{
Application of Geographic Information Systems and Asset Mapping to Facilitate Identification of Colorectal Cancer Screening Resources
}

\author{
Clement Kudzai Gwede ${ }^{1}$, Beverly G Ward ${ }^{2}$, John S Luque ${ }^{3}$, Susan T Vadaparampil ${ }^{1}$, \\ Desiree Rivers ${ }^{4}$, Dinorah Martinez-Tyson ${ }^{4}$, Shalewa Noel-Thomas ${ }^{1}$, Cathy D Meade ${ }^{1}$ \\ ${ }^{1}$ Moffitt Cancer Center, Department of Health Outcomes and Behavior, Division of \\ Population Sciences, Tampa, FL \\ ${ }^{2}$ BGW Associates, LLC, Tampa, Florida \\ ${ }^{3}$ Georgia Southern University, Jiann-Ping Hsu College of Public Health, Georgia \\ ${ }^{4}$ University of South Florida, Tampa, FL
}

\begin{abstract}
Objective: We sought to identify and map the geographic distribution of available colorectal cancer screening resources; following identification of this priority within a needs assessment of a local community-academic collaborative to reduce cancer health disparities in medically underserved communities.

Methods: We used geographic information systems (GIS) and asset mapping tools to visually depict resources in the context of geography and a population of interest. We illustrate two examples, offer step-by-step directions for mapping, and discuss the challenges, lessons learned, and future directions for research and practice.
\end{abstract}

Results: Our positive asset driven, community-based approach illustrated the distribution of existing colonoscopy screening facilities and locations of populations and organizations who might use these resources. A need for additional affordable and accessible colonoscopy resources was identified.

Conclusion: These transdisciplinary community mapping efforts highlight the benefit of innovative community-academic partnerships for addressing cancer health disparities by bolstering infrastructure and community capacity-building for increased access to colonoscopies. 
Application of geographic information systems (GIS) and asset mapping to

Facilitate identification of colorectal cancer screening resources

\section{Introduction}

Colorectal cancer is the third most common cancer and the third leading cause of cancer deaths in American men and women [1] with an estimated 146,970 new cases and 49,920 deaths expected in 2009. The mortality burden is greatest in African Americans/Blacks, the uninsured and other socio-economically disadvantaged groups. With regards to reducing disease burden, colorectal cancer screening is recommended for men and women over the age of 50 years in the U.S. Survival after a colorectal cancer diagnosis depends on a multitude of factors including the stage of disease at diagnosis. While five-year survival rates exceed $90 \%$ if disease is detected and treated in the early stages, only about 39\% of colorectal cancers are diagnosed in the early stages. High among the barriers to early detection is lack of access to affordable and acceptable screening resources for the general population, and especially for the uninsured and medically underserved communities [1].

The Institute of Medicine (IOM) and the Department of Health and Human Services (DHHS) have identified the need for relevant strategies to improve access to healthcare services and to support the elimination of health disparities [2-6]. Cancer health disparities result from the complex interplay of economic, social, behavioral and environmental circumstances that impact differential access. As Freeman relates, "Disease often occurs within the context of human circumstances, including social position, economic status, culture, and environmental context” [7]. Hence there is a growing literature documenting cancer related health disparities from a geographic perspective [3, 8, 9]. However, with few exceptions [10-13], not much geographic analysis has been done for colorectal cancer, a detectable, preventable and treatable malignancy characterized by marked racial-ethnic disparities.

In this paper, we share our experiences applying geographic information systems (GIS) and asset mapping to explore availability and accessibility of culturally responsive colorectal cancer screening services in the Tampa Bay Community Cancer Network (TBCCN) collaborative. We describe two examples of GIS and asset mapping among TBCCN partner organizations and service region, describe the methods of data capture and preparation for mapping, offer step-by-step directions for GIS and asset mapping, and present preliminary findings that have informed research and practice decisions related to accessing colorectal cancer screening and related services. The challenges, lessons learned and implications for other local or national cancer control programs seeking to map similar indicators for community engagement are also discussed.

\section{Contributions of GIS and asset mapping: Integrating new technology and traditional data tools}

An emerging technology in cancer health disparities research [3], GIS can be combined with another instrument, asset mapping to understand and visualize specific social and health conditions and resources that impact health disparities in the context of geography. GIS technology provides researchers, practitioners, policy makers and other decision makers the ability to integrate health data with mapping functions and allow for in-depth investigation, exploration, visualization, and modeling of health outcomes patterns [3, 14, 15]. Evidenced by its 
broad application in many sub-disciplines of public health, including understanding patterns of and planning HIV interventions, mapping injuries and environmental injustice, and mapping distribution of practitioners [8, 9, 16-26], GIS has benefited communities and moved the science of public health forward.

In addition, there is a growing GIS literature mapping cancer epidemiology data by race, ethnicity, gender, disease and healthcare access [3, 14, 15, 27-29]. The utility of GIS mapping technology is its versatility in the use of traditional health data (e.g., incidence, mortality, resources) allowing researchers to present them in more dynamic ways to varied audiences such as community research partners, decision makers, or other stakeholders or other interested general public constituencies. However, the application of GIS to understand local cancer screening resources is less common. Thus, it is important to provide examples of how GIS and asset mapping are employed as integral tools in the context a local community-academic collaborative seeking to increase access to cancer screening resources for medically underserved populations.

GIS uses data generated at many levels of society and geography and one method of gathering and organizing these data is the asset mapping method. Asset mapping (community resource mapping, environmental scanning, social mapping, youth mapping) is the process of cataloging and highlighting the positive resources (rather deficits) in a community [30-34] to create awareness of community resources, identify connections among community providers, and meet community needs. Thus, in this context, the use of GIS is intended to directly address the needs of TBCCN partners and to benefit the community.

Our application of GIS and asset mapping in a local community-academic partnership network, represents a unique and rich opportunity for analysis of salient data to inform decisions. The overall goal of the TBCCN is to create a solid, robust and sustainable core organizational network infrastructure through collaborations, partnerships and community-capacity building activities. Funded by NCI's Center to Reduce Cancer Health Disparities, TBCCN aims to address critical access, prevention and control issues that impact medically underserved, lowliteracy and low-income populations in selected areas of an ethnically diverse tri-county area which includes a growing number of foreign-born residents. Meeting this goal involves cancer awareness and education activities, the use of CBPR methods, and the creation of sustainable collaborations and partnerships that improve access and utilization of cancer screenings. Specifically, a formal assessment of the TBCCN partners [35] identified an unmet need to increase access and availability of colorectal cancer screening resources. Access and affordability to these beneficial screening resources was prioritized by the TBCCN membership, therefore setting the stage for the GIS and asset mapping efforts reported here.

In a related partnership, the Tampa-based Patient Navigator Research Program (PNRP) (U01 CA117281) also strives to address access and utilization of cancer screening services for the medically underserved. This study seeks to mitigate barriers and facilitate timely access to diagnosis and appropriate care using existing community resources. As such, both cancer health disparities collaborative programs benefit from the use of GIS and asset mapping to improve identification, accessibility and use of affordable colorectal cancer screening resources. 


\section{Methods}

Several factors informed our decision to consider the use of GIS and asset mapping to the investigation of colorectal cancer resources. First, colorectal cancer is among the top cancers diagnosed in both men and women in the Tampa Bay Area [36]. Second, the American Cancer Society identified colorectal cancer as a major priority and published a document to motivate the public and medical communities to eliminate the unnecessary suffering caused by colorectal cancer [37]. Third, our community partners recognized and related a significant gap in the availability of colorectal screening and diagnostic services in the area according to a partner needs assessment [35]. Finally, the need for locating and accessing colorectal cancer screening and related services was further reiterated by feedback from navigators and investigators from the PNRP study who documented longer waiting times and limited sources for receipt of colonoscopies as compared to mammographies for similar patient populations. Unlike for breast and cervical cancer, there is no state funded program for colorectal cancer screening and early detection for medically underserved in Florida.

Thus, TBCCN investigators sought to map specific geographic areas with population characteristics, resource users with resources or access points, and spatial locations of different types of resources e.g., hospitals providing colonoscopies. By integrating and analyzing information in this way, users can assess local socio-demographic characteristics, assess the supply of resources, generate ideas for coordination of resources, assess relationships between resources and resource users (e.g., high risk groups and medical service locations), and generate maps that illustrate these relationships in a visually compelling way. In order to accomplish this, we were guided by strategies for engaging community members in processes that were consonant with a CBPR theoretical perspective and participatory action research $[38,39]$ to effect sustainable, positive health change. Community member input was integral throughout our efforts as the problem was first identified, as the processes became defined, and as the results were found. Institutional Review Board (IRB) approval and consent of participants were obtained before initiating data collection.

\section{Step-by-step: How to “map” community assets}

Asset mapping begins with defining the community, including the geographic boundaries and target population (e.g., inner-city, racial/ethnic, elderly, persons with disabilities, medically underserved, or by specific cancer diagnosis). The next step should include discussions on how the information collected will be used. If, for example, the data will be used to create a community resource guide, there may be a need to discuss the distribution scope and plan resources for printing and updating the materials. The third step is to decide which assets will be identified. This may include building on existing data sources and identifying and modifying inventory tools for data collection. The process of asset mapping is made with and from individual and community data. Two research strategies may reduce health disparities: community participation and the use of geographic information systems [13]. When combined with community participation, geographic information systems approaches, such as the creation 
of resource or population maps that connect disease focused resources/assets and with community context, can catalyze action to reduce health disparities.

Resources of interest to TBCCN include providers, cancer screening facilities/services (e.g., colonoscopies, mammograms), cancer treatment programs (e.g., hospitals, cancer centers, other outpatient treatment facilities), and other cancer-related support services (navigators, support groups, hospice, transportation, lodging). All these can be identified and assessed for their feasibility and utility (e.g., fee structure, payment plans, accessibility, efficiency) before they are included on a list or database to be mapped or distributed.

With an understanding of the types of assets in communities, a plan is developed to collect the information. Some considerations include target dates for data collection, method of data collection, types of resources needed to collect the data, e.g., people, copying, database creation and entry, and facilitators.

Much of the data collection can begin with existing information resources that build on previous surveys or asset map-type activities. Lacking these resources, one can begin by using existing macro-level gray data, e.g., censuses, directories, etc. This helps to provide a rapid assessment of available resources. Reliable sources of gray data include

- City, county, and agency websites;

- Phonebooks and city directories;

- $\quad$ The Internet (Google map is a great resource);

- Local newspapers;

- Other inventories or databases including local, state and national (e.g. the state cancer registries, state vital statistics, U.S. census, National Cancer Institute and Centers for Disease Control and Prevention databases).

- Local libraries;

- Community partners’ databases.

Finally, the process and results should be evaluated. Major aspects of evaluation include identifying the most and least effective techniques and processes, the tasks that were completed, and the percent of the target population reached. The final step of evaluation includes assessing outcomes with respect to products created, community benefit and extent that individuals/agencies in the community were included in the process of mapping, additional financial resources acquired based on mapping process, and improvements in access to care or reduction in disparities.

For long-term sustainability, one central question is: How will changes be captured, inventoried, and the information disseminated? A well delineated, iterative process should be developed to facilitate adding new partners and assets/resources, removing non-functional resources from the databases, and production of updated products such as maps or resource guides for distribution to stakeholders. 


\section{Results}

\section{Example \#1: Mapping colonoscopy resources in TBCCN}

We began with a baseline inventory of the Black population in Hillsborough, Pasco, and Pinellas counties by census tract from the 2000 decennial census [40] as logical population of reference for this illustration. These data were put into a GIS program and projected to show the Black population as a percentage of the total population across the three counties. As shown in Figure 1, there is considerable variation among and between the counties in the distribution of the Black population. Hillsborough County had the largest Black population, nearly 15\% of county's total; Pasco County had the smallest, slightly more than two percent. Within the counties, the Black population was concentrated among relatively few census tracts, with some tracts in Hillsborough and Pinellas counties, ranging from 60 to 99\% Black.

We then took the dataset of TBCCN partners, matched or geocoded the partners' addresses, and projected the dataset in a layer over the already mapped Black population. Geocoding is the process by which non-spatial data (address files) are linked to geographic coordinates and converted to map data (points on a map showing address locations). Most commercial GIS software packages have the ability to geocode and numerous geocoding services are also available on the Internet. In this case, geocoding of partners' addresses provided an initial assessment of the target population's access to referral and other supportive services provided by TBCCN partners. Finally, we added a layer of the hospitals/facilities within the counties and a 25-mile radius. This dataset was created by the American Hospital Association and was included in the GIS software. It served as a potentially available pool of providers for colorectal cancer screening resources for the target population.

Building on the information from the rapid assessment showing all hospitals and facilities (Figure 1), the research team identified a need for colorectal cancer screening resources or referral guides for uninsured and underinsured residents in Hillsborough County as a starting point. 


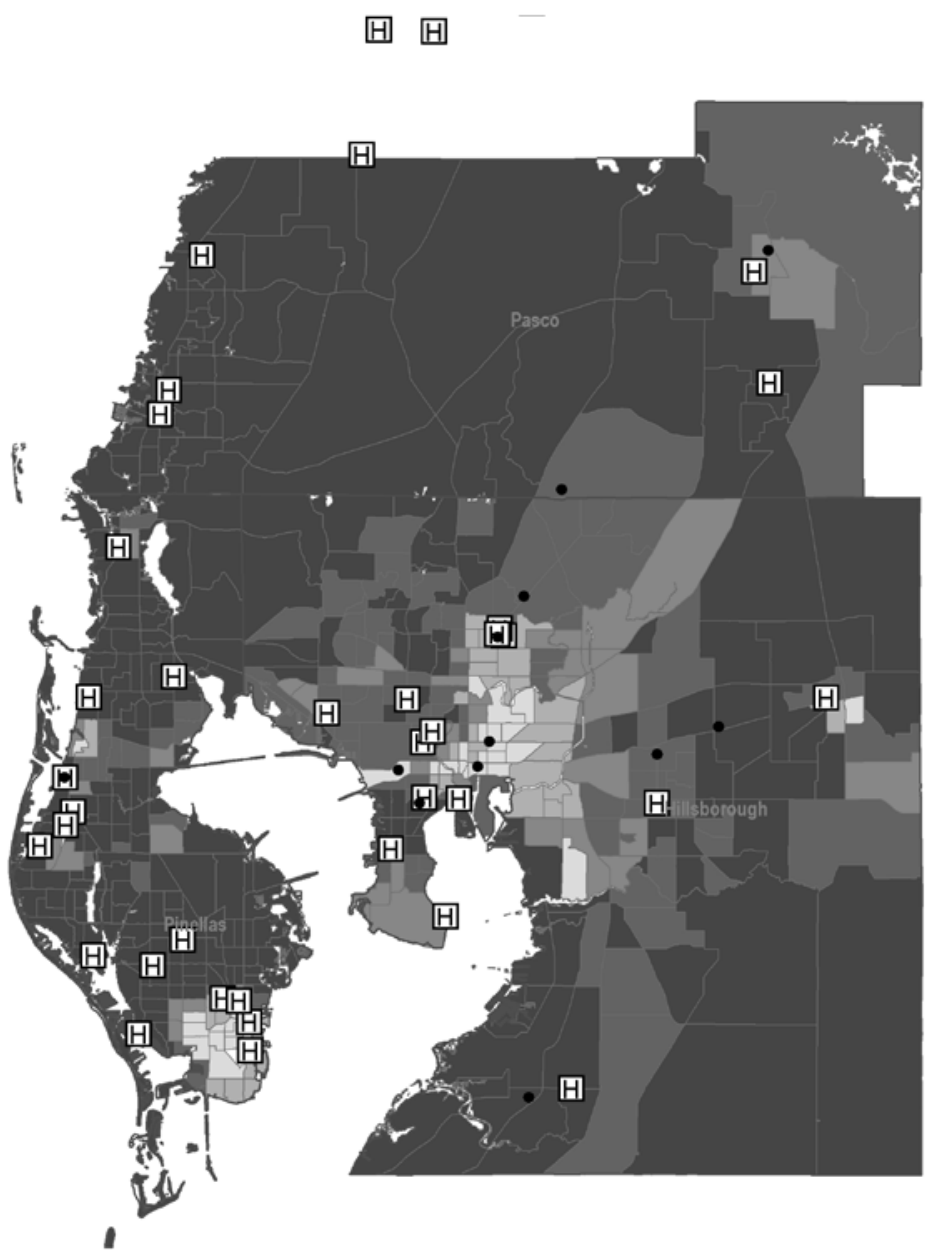

田

Figure 1. Black population, TBCCN community partners, and hospitals/facilities in three Florida Counties (Hillsborough, Pinellas and Pasco)

In addition to the potential hospitals, a Google ${ }^{\mathrm{TM}}$ search using the term "colorectal cancer screening" was conducted to identify other potential screening assets. However, colonoscopies are the most difficult to receive due to cost and facility requirements for this procedure. So we also searched Google TM using the term "colonoscopy". The results of the searches were downloaded into a table (Excel), imported into a GIS database, and geocoded. These assets were then projected over a map of the Black population of Hillsborough County. (See Figure 2.) 


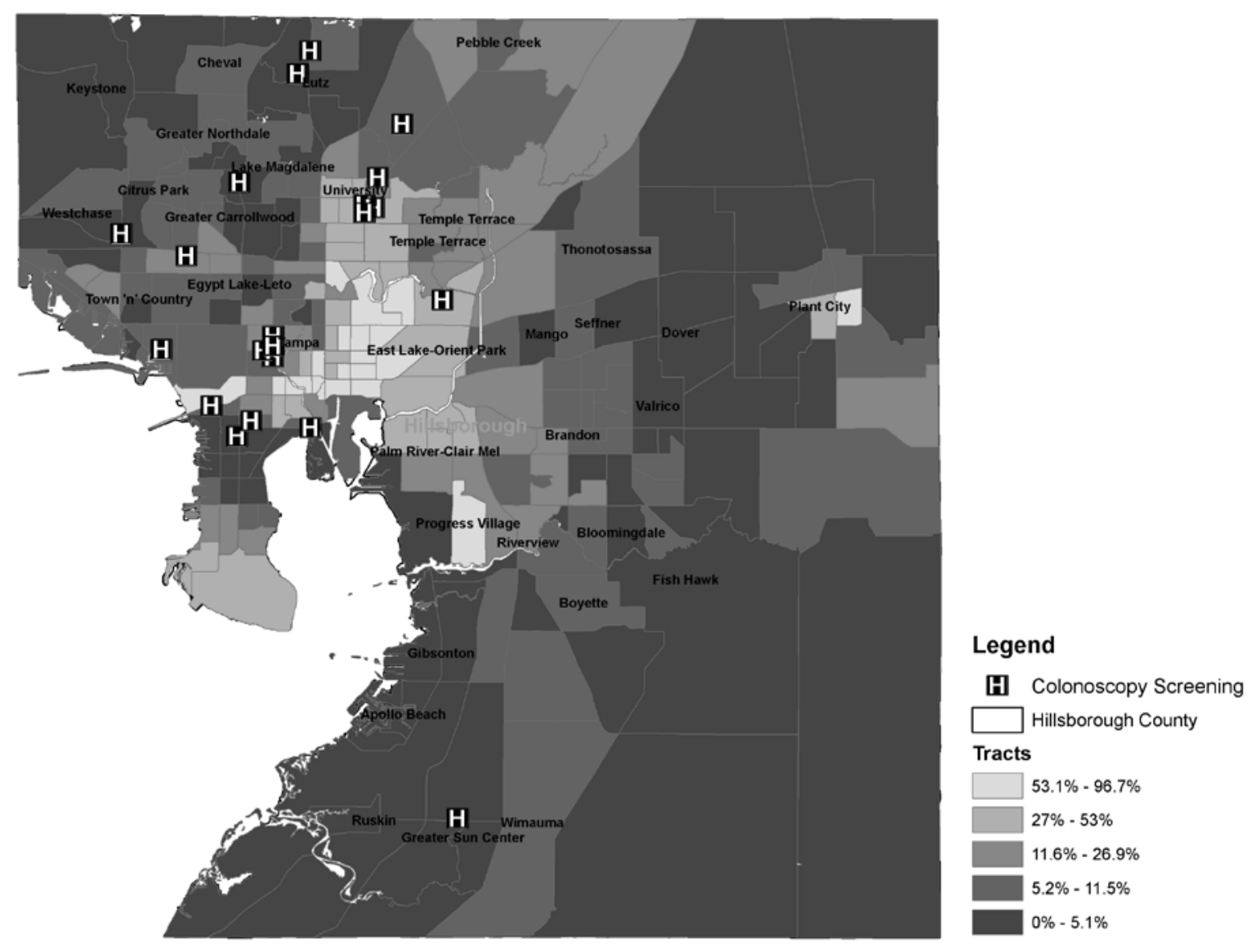

Figure 2. Colonoscopy hospital/facilities Google ${ }^{\mathrm{TM}}$ assets, Hillsborough County

Example \#2, pilot research project: "Colorectal cancer in ethnic subgroups of U.S. Blacks: Exploring disparities, U01 CA114627 S1.”

As part of the CNP competitive pilot grants mechanism, the "Colorectal cancer in ethnic subgroups of U.S. Blacks: Exploring disparities, U01 CA114627 S1” study was funded to explore perceptions of colorectal cancer in three subgroups of Blacks residing in medically underserved areas. Race-ethnicity was self-declared by participants. The long-term goal was to generate pilot data and set the stage for a future larger scale comparative or intervention study to increase colorectal cancer screening.

In this application, we geocoded the addresses of participants in the study and projected this layer over the map of the Black population and colonoscopy resources in Hillsborough County. (See Figure 3.) This application facilitated assessment of the geographic locations (neighborhoods) from which the sample was obtained relative to location of colonoscopy resources. This mapping also shows that the distribution of the sample doesn't differ by ethnic subgroup and geography. 


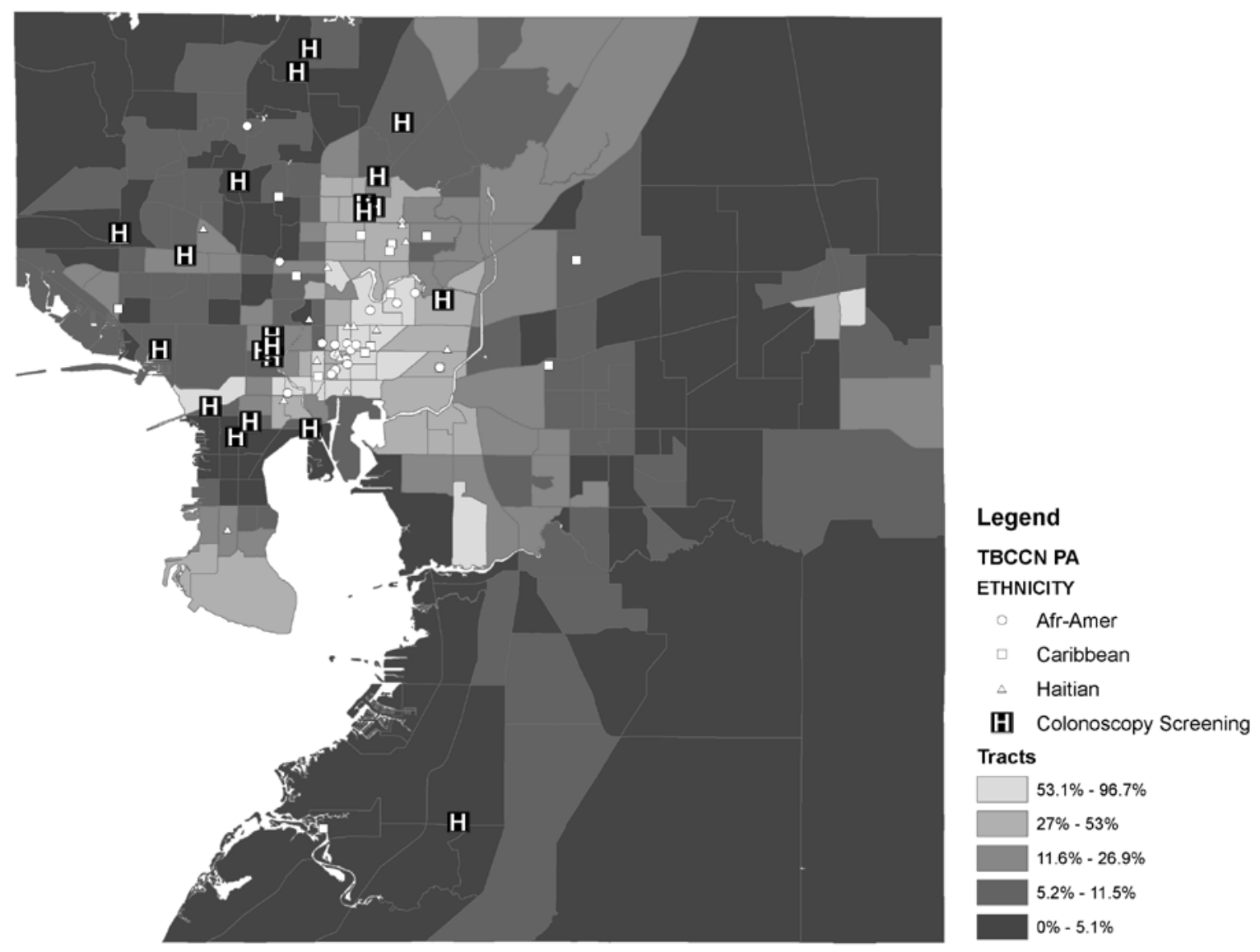

Figure 3. Enrollment to a TBCCN pilot study of three ethnic subgroups of Blacks, Hillsborough County

We also used data from the 2000 Census to identify by census tract concentrations of the Black population by ethnic subgroups, including sub-Saharan Africans, Caribbean Blacks, and Blacks 35 years of age and older. (See Figure 4.) 

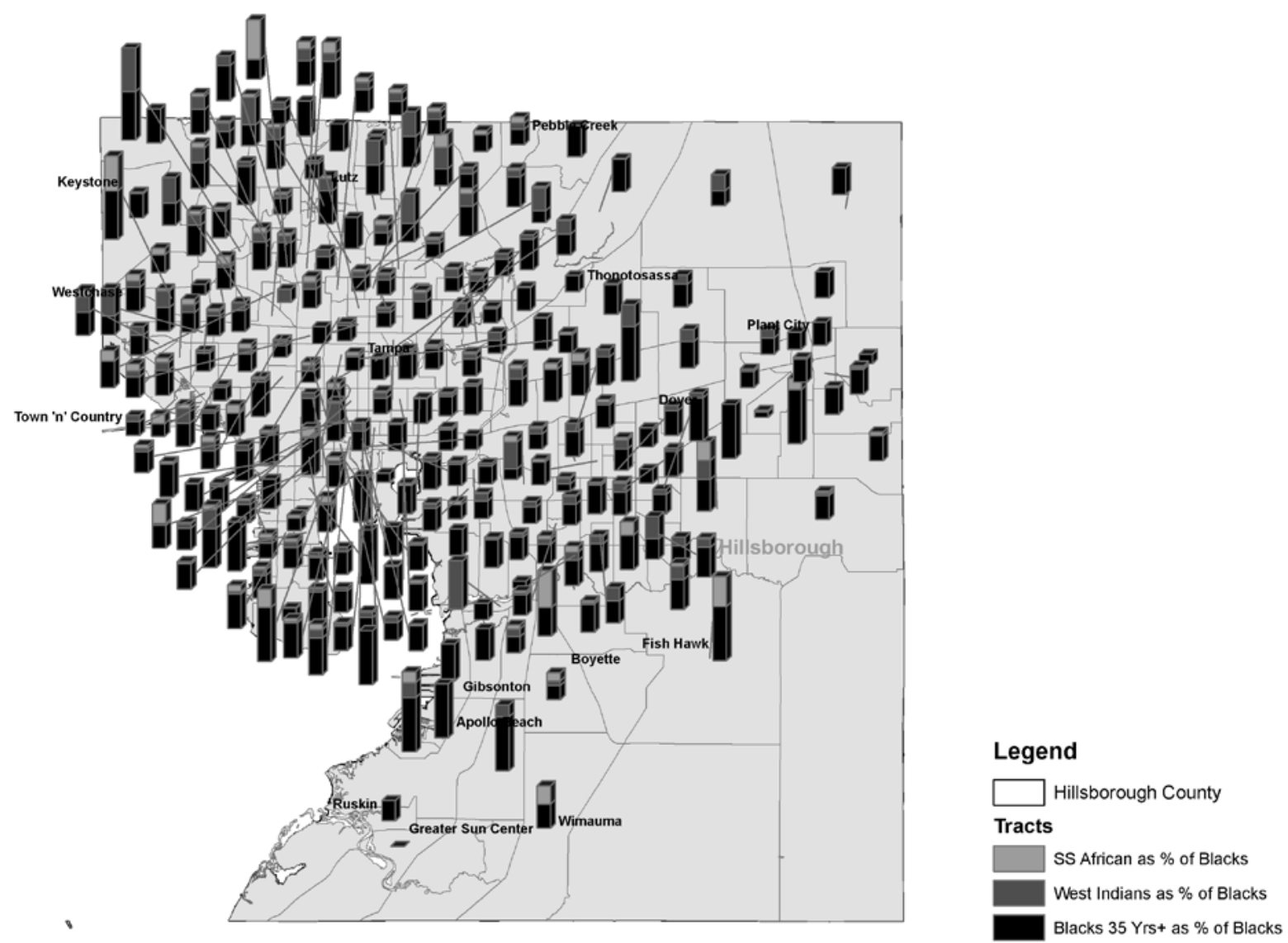

Figure 4. Black population by ethnic subgroups and age 35 years and older, Hillsborough County

The implications of these asset maps are significant. First, mapping of population and recruitment data informs our recruitment strategies for the future larger scale study. In addition, TBCCN researchers and community partners serving residents of historically underserved Black populations to gain a better appreciation of the diversity and location of these population subgroups.

\section{Discussion and conclusions}

\section{Challenges and lessons learned}

Our experience with GIS and asset mapping has facilitated several outcomes related to understanding and addressing health inequalities. Specifically, we identified resources available for colorectal cancer screening (assets) and identified a clear need to compile and present these assets in several ways to provide accessibility to community providers and members. Second, GIS also facilitated our understanding of the distribution of the Black population and pinpointed 
accrual to our pilot study on colorectal cancer. This provides the TBCCN with examples of how to combine multiple levels of data (population, cancer screening resources, and geographic distribution of study participants) using new and established tools, informed by a transdisciplinary team of researchers and community members. Ultimately, to be useful to the stakeholders, GIS and asset mapping should inform, improve understanding, and facilitate interventions that benefit the community directly in terms of service delivery and other ways of reducing inequalities in access to cancer care [13, 41].

The use of GIS and asset mapping initiatives provided us with notable challenges and lessons learned which may be helpful in transferring these methodologies to other settings. Three key challenges and lessons are discussed here: (1) finding accurate data sources to identify community assets (resources) for colorectal cancer screening; (2) developing the skills and capability to implement GIS in a meaningful way; and (3) involving community stakeholders in building, disseminating, and maintaining a useful database of the resources.

\section{Lesson \#1: Identifying community assets and accurate sources of data.}

Colorectal cancer screening resources were scarce particularly with regards to screening and diagnostic colonoscopies that are accessible and affordable for uninsured, or other medically underserved persons. As mentioned earlier our community assessment [35] identified many priority areas to address but it was important to narrow our scope and focus on one problem, one county at a time. Consequently, we focused on Hillsborough County, the largest of the three counties in population and area. We generated lists of colorectal cancer screening from many sources including resources available in the phone book, in Google ${ }^{\mathrm{TM}}$ search, and from our community partners based on prior relationships with these colonoscopy providers. A key challenge was that a large number of these assets needed to be verified to determine accuracy and accessibility in terms of fees and terms of payment, hours of service, and distance, for example. The academic partner assigned two staff members to call each identified colonoscopy provider, before retaining them on the list. Nevertheless, the CNP partners are committed to updating the list as information changes in the future.

\section{Lesson \#2: Developing skills to implement GIS in a meaningful way.}

For this part we developed a mutual collaboration with a GIS expert available at the university (BW). This individual had a strong interest in cancer control and a strong desire to reduce cancer health inequities having worked in related areas of health disparities and geography. This individual provided both technical and conceptual contributions. However, if one is not able to readily identify GIS expertise, there are many trainings available (search the Internet) that staff and investigators can complete. Two of the TBCCN co-investigators participated in a basic GIS workshop.

\section{Lesson \#3: Involving the community stakeholders in building, disseminating, and maintaining a useful database.}

Building and maintaining the resource databases and ensuring community benefit from the GIS mapping and related endeavors is a daunting task. It requires expressed ownership and committed participation by both the academic partners who provide the GIS/mapping expertise, and the community partners who use and have knowledge and 
experience with the availability, affordability, accessibility and functionality of the resources. Based on these lived experiences, the community partners can advise the academic partners on what changes to make to the resource directory and related GIS maps to maintain the accuracy and usefulness of this resource. Like in many other academic-community endeavors, time, flexibility and shared purpose ensure success in these collaborative often unfunded endeavors. The TBCCN collaborative is currently poised to participate in a number of policy initiatives to address statewide and national funding for colonoscopy screenings, diagnostic, and treatment resources for the uninsured. In partnership with a health policy advocate involved in the PNRP study, TBCCN is poised to share its mapping findings with state and federal legislators to bring awareness about the lack of these resources. Additionally, TBCCN is positioned to provide this information to state health agencies to support funding applications for service initiatives for colorectal cancer screenings similar to the Florida Breast and Cervical Cancer Early Detection Program for the medically underserved. In the meantime, our efforts are focused solely on completing mapping and evaluating its benefits. Our experience will certainly inform similar efforts of the challenges, how to best avoid pitfalls, and what benefits to expect.

\section{Mapping Future Directions}

Our application of GIS and asset mapping yielded asset maps that help users and practitioners to recognize and value the colorectal cancer screening resources within affected communities [42]. The pictorial representations of available resources (and inherent gaps) have facilitated discussions with resource managers and policy makers or redistribution of services that improve the health of medically underserved. Elimination of cancer health disparities is a national imperative [2-6, 13, 41] that requires integration of new and emerging technologies with other innovative perspectives not commonly employed in cancer control. Given, the availability of proven and beneficial early detection and treatment modalities for colorectal cancer and the heightened focus on attenuating cancer health disparities, the use of GIS and asset mapping in colorectal cancer screening represents a timely and innovative strategy in meeting the laudable goal of eliminating health inequalities. Moreover, it is important to recognize that communities have many assets--individual, associational, institutional and economic. With that said, more resources and funding for colorectal screening and other cancer-related services are needed in our collaborative and other communities nationwide.

Challenges to reducing inequalities and barriers to access are abundant. However, this process has provided baseline information to identify existing resources and to develop a resource guide for users and our partners. We have identified pertinent examples on using resources and GIS technology to improve our understanding of the inequitable access and derive data driven and informed solutions for reducing pervasive barriers to colorectal cancer screening. Similar methodologies may be employed to address barriers for other cancers and other health conditions locally, regionally or nationwide [2-5, 13, 41]. Future directions include more research and evaluation to better pinpoint community benefit, and identify the impact of GIS/resource mapping on access to screening and diagnostic colonoscopies to reduce related inequalities. 
Application of geographic information systems (GIS) and asset mapping to

Facilitate identification of colorectal cancer screening resources

\section{References}

[1] American Cancer Society, Cancer Facts \& Figures 2009. 2009, American Cancer Society: Atlanta. [2] Institute of Medicine, Unequal treatment: Confronting racial and ethnic disparities in health care ed. B.D. Smedley and A.R. Nelson. 2002, Washington, DC: National Academies Press.

[3] DHHS, Geographic information systems (GIS) and Cancer Research. 2008, Department of Health and Human Services, National Institutes of Health, National Cancer Institute: Bethesda, MD.

[4] Artinian, N.T., et al., Advancing the science of health disparities research. Ethn Dis, 2007. 17(3): 427-33.

[5] Tarlov, E., et al., Characteristics of mammography facility locations and stage of breast cancer at diagnosis in chicago. J Urban Health, 2009. 86(2): 196-213.

[6] Warnecke, R.B., et al., Approaching health disparities from a population perspective: the National Institutes of Health Centers for Population Health and Health Disparities. Am J Public Health, 2008. 98(9): 1608-15.

[7] Freeman, H.P., Poverty, culture, and social injustice: determinants of cancer disparities. CA Cancer J Clin., 2004. 54(2): 72-7.

[8] Bell, B.S., et al., Current practices in spatial analysis of cancer data: mapping health statistics to inform policymakers and the public. Int J Health Geogr, 2006. 5: 49.

[9] Pickle, L.W., et al., The crossroads of GIS and health information: a workshop on developing a research agenda to improve cancer control. Int J Health Geogr, 2006. 5: 51.

[10] Wang, F., Spatial clusters of cancers in Illinois 1986-2000. Journal of Medical Systems, 2004. 28(3): p. 237-56.

[11] Vieira, V., et al., Spatial analysis of lung, colorectal, and breast cancer on Cape Cod: an application of generalized additive models to case-control data. Environmental Health, 2005. 4: 11.

[12] Rushton, G., et al., Analyzing geographic patterns of disease incidence: rates of late-stage colorectal cancer in Iowa. Journal of Medical Systems, 2004. 28(3): p. 223-36.

[13] Beyer, K.M. and G. Rushton, Mapping cancer for community engagement. Preventing Chronic Disease, 2009. 6(1): p. A03.

[14] Graves, B.A., Integrative literature review: a review of literature related to geographical information systems, healthcare access, and health outcomes. Perspect Health Inf Manag., 2008. 5:

p. 11.

[15] Brewer, C.A., Basic Mapping Principles for Visualizing Cancer Data Using Geographic Information Systems (GIS). American Journal of Preventive Medicine, 2006. 30(2, Supplement 1): p. S25.

[16] Krieger, N., et al., Painting a truer picture of US socioeconomic and racial/ethnic health inequalities: the Public Health Disparities Geocoding Project. Am J Public Health, 2005. 95(2): p. 312-23.

[17] Maclachlan, J.C., et al., Mapping health on the internet: a new tool for environmental justice and public health research. Health Place, 2007. 13(1): p. 72-86.

[18] Olivet, M., et al., [Health services provision and geographic accessibility]. Med Clin (Barc), 2008. 131 Suppl 4: p. 16-22.

[19] Basara, H.G. and M. Yuan, Community health assessment using self-organizing maps and geographic information systems. Int J Health Geogr, 2008. 7: p. 67.

[20] Duncan, M.J., H.M. Badland, and W.K. Mummery, Applying GPS to enhance understanding of transport-related physical activity. J Sci Med Sport, 2009.

[21] Endacott, R., et al., Geographic Information Systems for healthcare organizations: a primer for nursing professions. Comput Inform Nurs, 2009. 27(1): p. 50-6. 
[22] Grinzi, P., A. Bazemore, and R.L. Phillips, Jr., Navigating general practice. The use of geographic information systems. Aust Fam Physician, 2008. 37(10): p. 855-8.

[23] Kazda, M.J., et al., Methodological complexities and the use of GIS in conducting a community needs assessment of a large U.S. municipality. J Community Health, 2009. 34(3): p. 210-5.

[24] Nelson, M.J., et al., A geospatial analysis of persons opting out of an exception from informed consent out-of-hospital clinical trial. Resuscitation, 2009. 80(1): p. 89-95.

[25] Vanmeulebrouk, B., et al., Open source GIS for HIV/AIDS management. Int J Health Geogr, 2008. 7: p. 53.

[26] Francois, F., et al., Colon cancer knowledge and attitudes in an immigrant Haitian community. J Immigr Minor Health, 2009. 11(4): p. 319-25.

[27] Krieger, N., et al., Race/Ethnicity and Changing US Socioeconomic Gradients in Breast Cancer Incidence: California and Massachusetts, 1978-2002 (United States). Cancer Causes Control, 2006. 17(2): p. 217-26.

[28] Xiao, H., et al., Analysis of prostate cancer incidence using geographic information system and multilevel modeling. J Natl Med Assoc, 2007. 99(3): p. 218-25.

[29] Hopfer, S., et al., Assessment of Training Needs and Preferences for Geographic Information Systems (GIS) Mapping in State Comprehensive Cancer-Control Programs. Health Promot Pract, 2008. 1: p. 1.

[30] Goldman, K.D. and K.J. Schmalz, "Accentuate the positive!": using an asset-mapping tool as part of a community-health needs assessment. Health Promot Pract, 2005. 6(2): p. 125-8.

[31] Goldman, K.D. and K.J. Schmalz, "As you likert it": conducting gap-based needs assessments. Health Promot Pract., 2007. 8(3): p. 225-8.

[32] Goldman, K.D. and K.J. Schmalz, Being well-connected: starting and maintaining successful partnerships. Health Promot Pract, 2008. 9(1): p. 5-8.

[33] Kretzmann, J. and J. McKnight, Building Communities from the Inside Out : A Path Toward Finding and Mobilizing a Community's Assets. , ed. N.I.N. Center for Urban Affairs and Policy Research, Northwestern University. 1993, Evanston, Ill; Chicago, IL: ACTA Publications [34] U.S. EPA, O.o.W., Community culture and the environment: A guide to understanding a sense of place. 2002, U.S. Evironmental Protection Agency.

[35] Author, et al., Strategies to Assess Community Challenges and Strengths for Cancer Disparities Participatory Research and Outreach. Health Promotion Practice, Online Frist. , 2009.

[36] FCDS. Florida Cancer Data System. 2009 [cited 2009 03/31]; Available from: http://fcds.med.miami.edu/inc/statistics.shtml.

[37] ACS, Colorectal Cancer Facts \& Figures 2008-2010. . 2008, American Cancer Society: Atlanta: GA.

[38] Greenwood, D.J., W.F. Whyte, and I. Harkavy, Participatory action research as a process and a goal. Human Relations, 1993. 46(2): p. 175-192.

[39] Israel, B.A., et al., Review of community-based research: assessing partnership approaches to improve public health. . Annu Rev Public Health 1998. 19: p. 173-202.

[40] United States Bureau of Census. Census 2000 summary file 1 (SF1). 2007 [cited 2009;

Available from:

http://factfinder.census.gov/servlet/DTGeoSearchByListServlet?ds name=DEC 2000 SF1 U\& lang =en\& ts=1972084495092007.

[41] Graves, A., A model for assessment of potential geographical accessibility: A csae for GIS. Online Journal of Rural Nursing and Health Care, 2009. 9(1): p. 46-54.

[42] Queralt, M. and A.D. Witte. A map for you? Geographic information systems in the social services. Social Work., 1998. 43: p. 455-14. 
doi: 10.5210/ojphi.v2i1.2893

Cite this item as: Gwede, C., Ward, B., Luque, J., Vadaparampil, S., Rivers, D., MartinezTyson, D., Noel-Thomas, S., \& Meade, C. 2010 Apr 9. Application of Geographic Information Systems (GIS) and Asset Mapping to Facilitate Identification of Colorectal Cancer Screening Resources. Online Journal of Public Health Informatics [Online] 2(1):e6. 